\title{
Retentissement des troubles cognitifs sur la vie sociale, professionnelle et émotionnelle des patients dans la forme adulte de la dystrophie myotonique de type I
}

\author{
Sabrina Sayah, Alexandre Morin
}

La forme adulte de la dystrophie myotonique de type I (DM1) est une maladie neuromusculaire qui, au-delà des symptômes somatiques, peut impacter les fonctions cognitives et plus spécifiquement les fonctions exécutives et la cognition sociale. Ces perturbations sont variables d'un individu à l'autre. Lorsqu'il existe une atteinte de la sphère exécutive et socio-émotionnelle, même discrète, elle peut avoir un retentissement important sur la vie sociale, professionnelle et émotionnelle des patients.

Sabrina Sayah Psychologue,

Neuropsychologue. Institut de Myologie, Centre de Référence de pathologies

Neuromusculaires

(Pr Bruno Eymard),

Groupe Hospitalier

Pitié-Salpêtrière, Paris,

France.

Alexandre Morin

Interne en DES

de Neurologie.

Institut de Myologie,

Centre de Référence

de pathologies

Neuromusculaires

(Pr Bruno Eymard),

Groupe Hospitalier

Pitié-Salpêtrière, Paris,

France.
La dystrophie myotonique de type I (DM1) est une pathologie neuromusculaire définie sur le plan génétique par une expansion de triplets dans le gène $D M P K$. En plus des symptômes musculaires, le phénotype comporte des atteintes multisystémiques liées à une expression génétique très variable selon les tissus. Dans la forme adulte, l'atteinte cérébrale est de découverte récente et serait à l'origine de troubles cognitifs chez certains patients [1, 2]. Il est fréquemment décrit des hypersignaux de la substance blanche dont les plus typiques sont au niveau des pôles temporaux (Figure 1). L'étude de Caso et al. recense des anomalies de la substance blanche significatives chez $95 \%$ des patients [3]. Des patterns diffus d'atrophie cérébrale de la substance grise au niveau des régions frontales, mais aussi temporales et pariétales, sont également mis en évidence (Figure 2) [4] en comparant les patients DM1 à des sujets contrôles à l'aide de la technique voxel base morphometry, permettant de comparer la densité de substance grise, voxel par voxel, dans les différentes régions cérébrales.

Au niveau moléculaire, l'expansion de triplet du gène DMPK est responsable de l'épissage anormal de certains gènes et donc de la production d'isoformes protéiques anormales. Il est ainsi retrouvé au niveau cérébral la présence d'une isoforme de Tau (protéines en lien avec certaines maladies neurodégénératives) spécifique à cette pathologie. Néanmoins, la corrélation entre les atteintes radiologiques et les atteintes cognitives n'a pas été démontrée. Un complément par imagerie de type tracking de fibres est prévu à l'Institut de la Mémoire et de la maladie d'Alzheimer (IM2A) et à
l'Institut de Myologie afin d'apporter de nouveaux éléments de réponse sur les mécanismes cérébraux sous-jacents à ces troubles. Les atteintes cognitivo-comportementales décrites dans cette pathologie peuvent avoir un retentissement conséquent sur la qualité de vie et sur l'insertion socio-professionnelle des patients [5]. Il paraît donc important, à la lumière de la littérature actuelle et des nouvelles données collectées dans le cadre du suivi habituel des patients à l'Institut de Myologie et lors d'une recherche effectuée à l'IM2A [6], de détailler les fonctions atteintes et les moyens de les prendre en charge.

\section{Les fonctions exécutives}

Les troubles cognitifs observés dans la DM1 concernent principalement les fonctions frontales et sont caractérisés par des difficultés exécutives et des troubles de la cognition sociale [2]. Les fonctions exécutives interviennent lorsque l'individu doit s'adapter à une situation nouvelle et que les schémas automatiques et routiniers ne sont plus suffisants. Elles permettent également d'adapter et de contrôler son discours, ses pensées et sa manière de répondre à une situation donnée. Les atteintes exécutives les plus fréquemment décrites chez les patients atteints de DM1 concernent la flexibilité mentale, l'inhibition et l'initiation. La planification, l'élaboration de stratégies et les capacités de mémoire de travail apparaissent quant à elle préservées [6].

L'inhibition permet de mettre de côté le schéma habituel afin de s'adapter à une situation nouvelle tandis que la flexibilité permet de passer d'une tâche à l'autre en fonction du but à atteindre. Des 


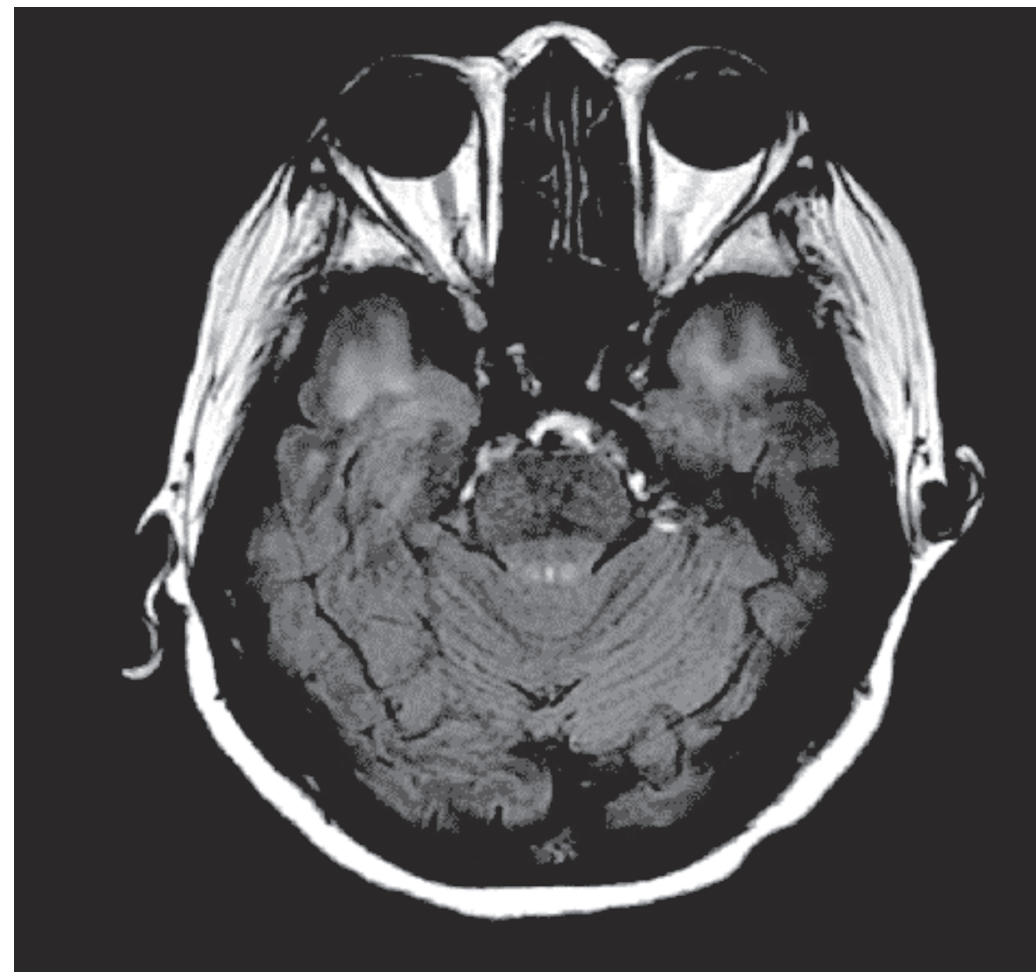

Figure 1

IRM cérébrale en T2 FLAIR : hypersignaux de substance blanche confluents des deux pôles temporaux.

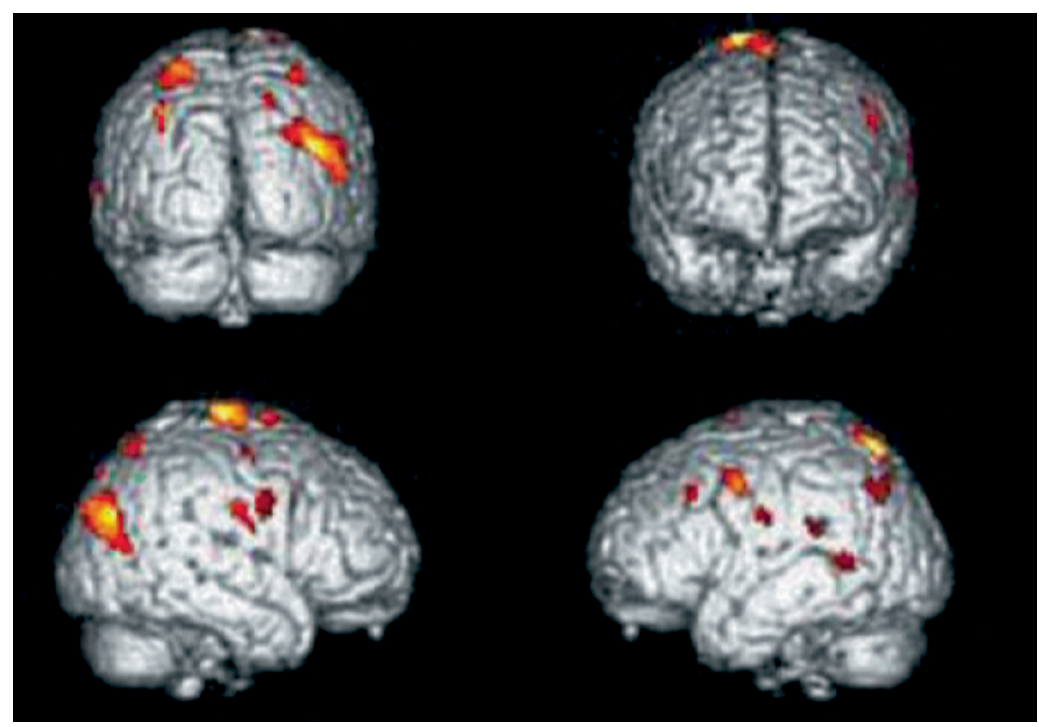

Figure 2

Patterns d'atrophie de substance grise en Voxel Base Morphometry chez les sujets DM1 adultes comparés à une population contrôle [4] (avec l'aimable autorisation de Antonini Get al. 2004, NESMOS Department, University of Rome Sapienza)

difficultés d'inhibition et de flexibilité peuvent entraîner des conduites dites "persévératives": le patient peut ainsi rester focalisé sur une seule et même idée pendant un long moment, reflétant non pas une personnalité rigide mais un défaut de flexibilité et d'inhibition. Il est également fréquent d'observer des conduites impulsives et une tendance à interrompre l'interlocuteur pendant son discours. Ces difficultés peuvent pénaliser le patient dans sa vie professionnelle et sociale et entraîner des situations de rejet ou d'incompréhension de la part des autres. Certains patients peuvent présenter une réduction des comportements volontaires dirigés vers un but. Cette apathie est caractérisée par une difficulté à initier, planifier et maintenir un comportement moteur, une idée ou une stratégie. L'entourage perçoit souvent ce manque d'initiative comme un trait de caractère et il leur arrive de qualifier à tort leur proche de " paresseux ", alors que ce comportement est le reflet d'une atteinte cognitive.

\section{La cognition sociale}

En ce qui concerne la cognition sociale, définissant l'ensemble des aptitudes et expériences émotionnelles et sociales régulant les relations entre les individus et permettant d'expliquer et prédire les comportements humains individuels ou en groupe [7], celle-ci peut être altérée notamment dans le champ de la théorie de l'esprit. La théorie de l'esprit fait référence à la capacité de se représenter et d'attribuer des états mentaux, des émotions et des intentions à soi-même et à autrui. Elle permet à l'individu de se décentrer de ses propres états mentaux afin de se mettre à la place de l'autre et de comprendre que certaines croyances ou désirs puissent différer d'une personne à l'autre. Des troubles de la théorie de l'esprit provoquent des situations sociales embarrassantes, des quiproquos ou des difficultés à comprendre l'ironie et le second degré. Les patients atteints de DM1 peuvent être gênés dans l'élaboration d'un sens sous-jacent à une situation sociale, un discours, une blague et par conséquent rencontrer des difficultés relationnelles importantes. Dans le milieu professionnel cette difficulté peut constituer un véritable obstacle par exemple lorsque le patient ne perçoit pas le mécontentement de son supérieur hiérarchique et persiste dans des comportements inadaptés à la situation créant ainsi un malaise dans la communication.

Il semble essentiel de noter que tous les patients ne présentent pas ces difficultés cognitives et qu'elles sont très variables d'un individu à l'autre en termes d'intensité et de répercussions dans la vie quotidienne. Quand ces troubles sont présents, ils peuvent amener à penser que les patients atteints de cette pathologie présentent une personnalité type. Il a d'ailleurs été montré que les patients atteints de DM1 présentent un profil de personnalité spécifique caractérisé par une baisse de l'expressivité émotionnelle. Ce déficit émotionnel peut être consécutif à une 
réaction d'adaptation à la maladie et/ou au retentissement de l'atteinte des fonctions cognitives [8].

\section{L'apport du bilan neuropsychologique}

Compte tenu de l'impact de ces troubles sur la vie sociale, professionnelle et émotionnelle des patients, un bilan neuropsychologique centré sur les fonctions frontales peut être demandé dans le but d'investiguer la présence de ces troubles et d'en informer le patient et son entourage familial. La restitution du bilan peut alors permettre de débloquer de nombreuses situations d'incompréhension pouvant exister dans certaines familles. Une restitution psychoéducative constituerait également un moyen d'encourager l'entourage à attribuer certains comportements spécifiques à ces troubles cognitifs et non à un aspect négatif de la personnalité du patient. À terme, une prise en charge cognitive adaptée pourra être proposée.

Une recherche multicentrique est réalisée dans différents services hospitaliers prenant en charge des patients atteints de DM1. Cette étude, appelée OPTIMISTIC, se base sur une approche psychothérapeutique cognitivo-comportementale (TCC) [9] centrée sur les difficultés rencontrées dans la vie quotidienne dans le but de limiter les comportements, les pensées et les représentations qui en sont à l'origine. L'objectif est d'améliorer la qualité de vie des patients en se basant sur trois difficultés majeures retrouvées dans cette pathologie : la fatigue intense, la réduction d'initiative et les difficultés relationnelles. Ce protocole en cours a pour visée de prendre en compte ces difficultés d'ordre cognitif afin de les inclure dans une approche thérapeutique plus globale. Enfin, il est important de rappeler que le patient a une histoire de vie avec des problématiques propres ; les prises en charge se doivent alors d'être adaptées à chaque patient en fonction de ses besoins et demandes.

\section{En conclusion}

Il semble nécessaire, au vu des connaissances actuelles, d'intégrer l'évaluation et la prise en charge des troubles cognitifs au parcours de soin des patients atteints de DM1. La mise en place d'un protocole d'imagerie avec tracking de fibres permettra à l'avenir d'apporter de nouveaux éléments de réponse sur les liens entre les mécanismes cérébraux et les troubles cognitifs dans cette pathologie. Cette technique utilisant l'IRM de diffusion permet d'analyser la densité des faisceaux de substance blanche selon leur localisation et d'évaluer leur intégrité. Une meilleure compréhension des manifestations cérébrales amènera certainement à une meilleure gestion de ces troubles.

Impact of cognitive impairment on the social, professional and emotional life of adult patients with myotonic dystrophy type 1

\section{LIENS D'INTÉRÊT}

Les auteurs déclarent n'avoir aucun lien d'intérêt concernant les données publiées dans cet article.

\section{RÉFÉRENCES}

1. Meola G, Sansone V, Perani D, Scarone S, Cappa S, Dragoni C, Cattaneo E, Cotelli M, Gobbo C, Fazio F, Siciliano G, Mancuso M, Vitelli E, Zhang S, Krahe R, Moxley RT. Executive dysfunction and avoidant personality trait in myotonic dystrophy type 1 (DM-1) and in proximal myotonic myopathy (PROMM/DM-2). Neuromuscul Disord 2003 ; 13 : 813-21.

2. Meola G, Sansone V. Cerebral involvement in myotonic dystrophies. Muscle Nerve 2007 ; 36 : 294-306.

3. Caso F, Agosta F, Peric S, Rakočević-Stojanović V, Copetti M, Kostic VS, Filippi M. Cognitive impairment in myotonic dystrophy type 1 is associated with white matter damage. PLoS One 2014 9: e104697.

4. Antonini G. Cerebral atrophy in myotonic dystrophy: a voxel based morphometric study. J Neurol Neurosurg Psychiatry $2004 ; 75$ : 1611-3.

5. Antonini G, Soscia F, Giubilei F, De Carolis A, Gragnani F, Morino S, Ruberto A, Tatarelli R. Health-related quality of life in myotonic dystrophy type 1 and its relationship with cognitive and emotional functioning. $J$ Rehabil Med 2006 ; 38 : 181-5.

6. Morin A. Étude des troubles cognitifs et comportementaux de la dystrophie myotonique de type 1 . Thèse de médecine, Université Paris Descartes, 2015.

7. Allain P, Aubin G, Gall DL. Cognition sociale et neuropsy chologie. Bruxelles: De Boeck, 2012 : 400 p.

8. Bungener C, Jouvent R, Delaporte C. Psychopathological and emotional deficits in myotonic dystrophy. $J$ Neurol Neurosurg Psychiatry 1998 ; 65 : 353-6.

9. Van Engelen B. Cognitive behaviour therapy plus aerobic exercise training to increase activity in patients with myotonic dystrophy type 1 (DM1) compared to usual care (OPTIMISTIC): study protocol for randomised controlled trial. Trials $2015 ; 16: 224$. 